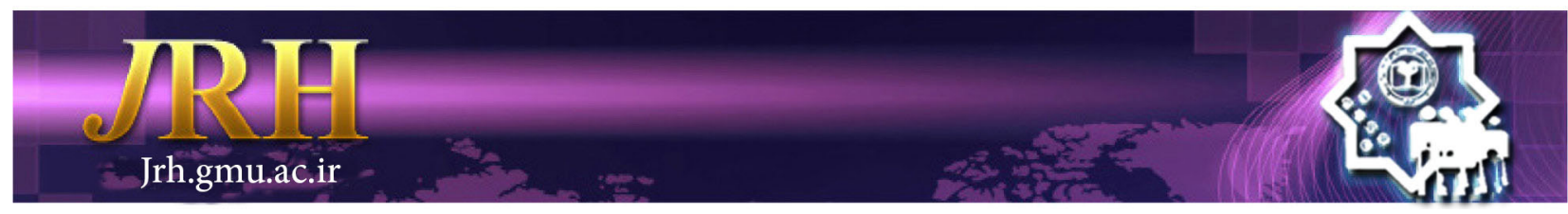

\title{
Effect of music on postoperative physiological parameters in patients under open heart surgery
}

Neda Mirbagher Ajorpaz ${ }^{1}$, Abouzar Mohammadi², Hamed Najaran², Shala Khazaei ${ }^{2}$

Journal of Research \& Health

Social Development \& Health Promotion Research Center

Vol. 9, No.3, May \& Jun 2019

Pages: 195- 202

DOI: 10.29252/jrh.9.3.195

Original Article

1. Correspondence to: Department of operating Room,School Nursing and Midwifery, Autoimmune Diseases Research Center, Kashan University of Medical Sciences, Kashan, Iran

Email: mirbaghern@ kaums.ac.ir

2. Department of Operating Room, School Nursing and Midwifery, Trauma Nursing Research Center, Kashan University of Medical Sciences, Kashan, Iran

Received: 8 May 2014

Accepted: 18 Oct 2014

How to cite this article: Mirbagher Ajorpaz N, Mohammadi A, Najaran H, Khazaei Sh. Effect of music on postoperative physiological parameters in patients under open heart surgery. J Research \& Health2019; 9(3): 195- 202.

\begin{abstract}
Music as a therapeutic non-invasive tool-could be used as an effective intervention in nursing practice. The aim of this study was to survey effect of music upon patients' physiological parameters following open heart surgery. A quasi-experimental study was performed on 60 patients under open heart surgery referred to intensive care unit. Patients were randomly divided into two groups including experimental and control groups. Patients in experimental group listened to non verbal music for 30 minutes after surgery by headphones. The control group did not receive any intervention. Before and after intervention, physiological parameters was measured in two groups. A significant difference was demonstrated in pulse rate, systolic blood pressure and oxygen saturation of experimental group's patients. But there was no significant difference in respiratory rate and diastolic blood pressure. As for music would reduce some of the physiological parameters, and have some advantages, it could be suggested to use for controlling physiological parameters following surgery.
\end{abstract}

Keywords: Blood Pressure, Music, Oxygen Saturation, Pulse, Respiratory

\section{Introduction}

Cardiovascular diseases have the highest death rates and will remain the primary cause of death in the world until 2020. Nearly 52 percent of deaths in the United States and 48 percent in Europe are related to these diseases [1].

A large number of patients with coronary artery diseases which do not respond to medical therapies, have to undergo coronary artery bypass graft surgery [2]. This common procedure has been done for about 35 years [2]. The prevalence of coronary artery bypass graft surgery is $26.79 \%$ in North America, $0.72 \%$ in Asia, $17.94 \%$ in Western Europe and $18.14 \%$ in the rest of the world. In Iran, $60 \%$ of all open-heart surgeries are coronary artery bypass surgeries [3] .

Most of the patients were taken to operating theatre, experience different levels of anxiety and fearing. The first target of emotional attacks is cardiovascular system which even mild stresses causing cardiovascular symptoms to be appearing. The heart is one of the organs that react to stress and anxiety [1,2] and physiological signs of these reactions appear with hypertension, highpulse and respiratory rates [3]. Music therapy is a simple- and cheap method to reduce stress and psychological changes. The prevalence 
of cardiovascular diseases has been increased during the last 2 centuries. The disease has been known as a main cause of morbidity and mortality right across the globe [4]. 300000 patients would die of cardiovascular diseases, in Iran yearly [5]. Surgery is described as a stressor factor, and also causes psychological (anxiety and fearing) and physiological (endocrine responses) reactions. More expansion of surgery would provide more physiological changes [6]. The nature of cardiac surgery needing admission, hospitalizing for a 1 to 3 days period in Intensive Care Unit (ICU) [7] this ward is a stressor ambience owing to clinical and/or surgical therapeutic circumstances and environmental factors, in addition, the patients hospitalized in such wards have to stand emotional and psychological displeasures [8]. Consequently, physiological responses to stress, the levels of such hormones such as; Epinephrine and Nor-Epinephrine hormones, would be increased and this causes tachycardia and also increasing of blood flow to person's muscles, the vital signs would be higher than normal level, in the following, which could provide some risks for the patient such as needing more oxygen to the muscles. The hypertension could tear the sutures line after cardiac surgery, also, increase in bleeding after the surgery, and even rupturing in graft's anastomosis. In addition, post-surgical pain resulted from sternum bone incision and being of chest-tube could cause superficial respiration in the patient, and also could be a barrier in coughing and deep breath which influence upon respiratory system function, finally. The function of patients' respiratory system should be appraised by Arterial Blood Gas (ABG) test and pulse oximetery in order to lower stress effects of hospitalized patients in Intensive Care Unit (ICU), and also psychological- and physical problems and preventing sympathetic reactions during the spontaneous breath [9].

Using palliative techniques such as listening to tranquil-music, directed-mind imagination, massage, and so on is an effective tool to reduce stress and fixing vital signs in normal level [8]. Using music as a therapeutic tool back to previous civilizations, as Greece-, Egypt-, China-, India-, and Rome epigraphs has mentioned it as a healing mean [10]. The music could be used as an effective intervention in nursing practice as a part of patient's care program and used as a therapeutic non-invasive tool in order to palliate pain, increasing sooth and strengthening body immune system [11]. Music is an inexpensive, non-pharmacological, noninvasive nursing interaction that has no side effects that can be effective along with other methods $[2,6]$. Although music effect mechanism in reducing the physiological parameters is not clear completely, increasing of receptors on the cells surface and increasing of Endorphins are said as the probable mechanisms in this context [11].

Publishing more articles prove musical therapeutic favorable effects and admitting by people in the 20 century [12]. There was a significant difference in physiological parameters of immature neonates via music therapy, the results of Alipoor et al. study pointed out [13]. Vaajoki et al. illustrated that music therapy cause a significant difference in some of the physiological parameters such as systolic blood pressure [14]. Camara et al. indicated that music therapy would lower all of the physiological parameters in ophthalmic-under-surgery patients [15]. There is a significant correlation between listening to music with anxiety level and controlling vital signs of patients, the results of Labrague study indicated [16]. The result of Ghetti study which was performed upon patients under cardiac catheterization confirms the mentioned consequences [17]. There was no significant influence upon controlling vital signs of cataract-undersurgery elders, Cruise et al. illustrated [18].

There are low studies on the effect of music on physiological parameters after open heart surgery. In addition, music has a cultural implication and insufficient studies have been conducted in countries such as Iran. Meanwhile, the success of music therapy may be greatly enhanced by 
determining patient's preference, familiarity and cultural context. While some studies have demonstrated physiological parameters to decrease after music, others found music to be ineffective on physiological parameters .Many previous investigations have been limited in a way. For instance, some employed a small sample size, some evaluated different types of surgeries and anesthesia in one study, and some used a type of music not selected by patients. Moreover, while a systematic review recommended a minimum duration of 30 minutes for music therapy to be effective in clinical practice, a number of studies played music for less than 30 minutes. On the other hand, the constant presence of the researcher during the intervention might have affected patient's response. Therefore, considering the above mentioned facts and according to the cultural, social and economic differences in Iran, we tried to perform this study.

The aim of this study was to investigate the effect of music on physiological parameters in patients under open heart surgery.

\section{Method}

A quasi-experimental study was performed on 60 patients under open heart surgery referred to ICU of the hospital in Kashan city, Southeast of iran. The inclusion criteria were age between 18 to 60 years, complete conciseness, reading and writing ability, first time open heart surgery, Right-handed patients, Lack of endotracheal tube and chest tube, Lack of erythema. Patients were excluded if they were connected to a ventilator at the time of intervention, chronic pain, audition decreasing, some problems during measuring and hemodynamic instability.

The sample size in each group was determined based on the following assumptions: power $=0.80, \alpha=0.05$, the minimum expected difference in standard deviation $=3.6$, and the minimum expected difference in means to be 2.40 [8]. According to the formula, the sample size in each group was 30 members. The data collection instrument consisted of two parts; first part was the demographic characteristics including age, gender, marital status, surgery type, education level, and the second part included Physiological parameters include systolic- and diastolic blood pressure, pulse rate, oxygen saturation $\left(\mathrm{SPO}_{2}\right)$ and respiratory rate which were measured by Nonin $^{\mathrm{TM}} 7500$ Pulse Oximeter, Finland. Systolic and diastolic blood pressure, heart rate, respiratory rate, $\mathrm{SPO}_{2}$ were measured by heart monitor .

After explaining the research objectives and obtaining written informed consent from the participants. The data were collected between 3.00 p.m. and 4.00 p.m. when the traffic in ICU was not intense and the patients were not receiving invasive or noninvasive procedures. All of the patients received standard cares under supervision of a cardiovascular specialist. All the patients received $50 \mathrm{mg}$ Pethidine and beta- receptor blocker (metoprolol succinate) at 8 am by the ICU nurse. The patients also received 3-5 $\mathrm{ml} / \mathrm{min}$ oxygen delivered by nasal cannula to prevent complications and hypoxemia after surgery. The second researcher helped the participants lie down in the bed and turn over to a relaxing position on their back, supine position, with the bed head at 20 to 30 degrees. In addition, factors affecting pain intensity, such as incision method, type and extent of incision, having chest tube, and type of analgesic drugs used, were the same for all patients. Demographic and Physiological parameters $\left(\mathrm{SPO}_{2}\right.$ and respiratory rate) were evaluated in the first 24 hours after the surgery in the ICU. The patients in the music group were listening to sedative music by an MPEG 1 Layer 3 (MP3) player with special headphones for 30 minutes. Sedative music was operationalized as music without lyrics and with a sustained melodic quality, with a rate of $60-80$ beats per minute and a general absence of strong rhythms or percussion. Changing the volume was in the control of the patient. Sedative music was selected by a music expert considering the cultural conditions of the society. During the intervention, for all participants, the 
environment was enhanced to reduce stimuli and facilitate rest by closing the door and posting a sign to prevent being disturbed by visitors and health care personnel.

During this period, the second researcher stayed in ICU. After the intervention, $\mathrm{SPO}_{2}$ and respiratory rate was again evaluated immediately after the music was completely played in two groups. The data of control group were collected without any force to listening music in the same intervals like experimental group.

In order to analyze the data, SPSS-16 and descriptive-analytical tests were used. For determining demographic characteristics, descriptive statistics and for Physiological parameters analytical tests (t-test) was used.

\section{Results}

A total number of 60 patients undergoing open heart surgery were enrolled in this study ( 30 in each group). A proportion of $43.3 \%$ of patients were in the age range of 50-65 years old. Also, $56.6 \%$ of the patients were male and all married (Table 1).

Table 1 Demographic data of experimental and control groups

\begin{tabular}{|c|c|c|c|c|}
\hline \multicolumn{2}{|c|}{ Variable } & $\begin{array}{l}\text { Experimental } \\
\text { group }\end{array}$ & Control group & Sig. \\
\hline \multirow{2}{*}{ Gender } & Female & $18(60 \%)$ & $13(43.3 \%)$ & \multirow{2}{*}{$\begin{array}{c}\text { Chi-square }=3.51 \\
p<0.1\end{array}$} \\
\hline & Male & $12(40 \%)$ & $17(56.6 \%)$ & \\
\hline \multirow{2}{*}{$\begin{array}{l}\text { Marital } \\
\text { status }\end{array}$} & Married & $25(83.3 \%)$ & $23(76.6 \%)$ & \multirow{2}{*}{$\begin{array}{c}\text { Chi-square }=2.86 \\
\mathrm{p}<0.09\end{array}$} \\
\hline & Single & $5(16.6 \%)$ & $7(23.3 \%)$ & \\
\hline \multirow{3}{*}{ Age group } & $20-35$ & $6(20 \%)$ & $8(26.6 \%)$ & \multirow{3}{*}{$\begin{array}{c}\text { Chi-square }=3.11 \\
\mathrm{p}<0.28\end{array}$} \\
\hline & $35-50$ & $11(36.6 \%)$ & $8(26.6 \%)$ & \\
\hline & $50-65$ & $13(43.33 \%)$ & $14(46.6 \%)$ & \\
\hline \multirow{3}{*}{$\begin{array}{l}\text { Education } \\
\text { level }\end{array}$} & $\begin{array}{l}\text { Secondary } \\
\text { education }\end{array}$ & $11(36.6 \%)$ & $9(30 \%)$ & \multirow{3}{*}{$\begin{array}{c}\text { Chi-square }=3.9 \\
\mathrm{p}<0.14\end{array}$} \\
\hline & Diploma & $9(30 \%)$ & $14(46.6 \%)$ & \\
\hline & Academic & $10(33.33 \%)$ & $7(23.3 \%)$ & \\
\hline \multirow{2}{*}{$\begin{array}{l}\text { Type of } \\
\text { surgery }\end{array}$} & $\begin{array}{l}\text { Valve } \\
\text { replacement }\end{array}$ & $10(33.3 \%)$ & $8(26.6 \%)$ & \multirow{2}{*}{$\begin{array}{c}\text { Chi-square }=2.91 \\
\mathrm{p}<0.15\end{array}$} \\
\hline & CABG & $20(66.6 \%)$ & $22(73.33 \%)$ & \\
\hline
\end{tabular}

No significant difference was observed between the mean of Physiological parameters in the experimental and control groups before intervention $(p \geq 0.05)$ However, a significant difference was found between the mean of systolic blood pressure and pulse rate in the experimental and control group at 30 minutes after music therapy $(p<0.03$ and $p<0.04$, respectively). But the significant difference was not indicated in mean of diastolic blood pressure in the two groups after music therapy $(\mathrm{p}<0.08)$ (Table 2).

Table 2 Comparing mean of physiological parameters in each group before and after intervention

\begin{tabular}{lcccccc}
\hline \multirow{2}{*}{ Physiological parameters } & \multicolumn{3}{c}{ Experimental group } & \multicolumn{3}{c}{ Control group } \\
\cline { 2 - 7 } & Before & After & p-value* & Before & After & p-value* \\
\hline Systolic blood pressure & $140.1 \pm 3.18$ & $120.2 \pm 2.1$ & 0.05 & $150.2 \pm 2.8$ & $145.3 \pm 1.2$ & 0.8 \\
Diastolic blood pressure & $110.2 \pm 0.28$ & $100.2 \pm 0.8$ & 0.06 & $95.2 \pm 1.4$ & $92.1 \pm 1.8$ & 0.28 \\
Pulse rate & $110.1 \pm 2.3$ & $82.3 \pm 2.2$ & 0.02 & $115.1 \pm 4.2$ & $1.8 .2 \pm 3.2$ & 0.09 \\
Respiratory rate & $24.2 \pm 1.2$ & $22.3 \pm 1.8$ & 0.01 & $28.2 \pm 3.1$ & $24.2 \pm 2.2$ & 0.06 \\
$\mathrm{SPO}_{2}$ & $96.3 \pm 2.2$ & $97.7 \pm 2.1$ & 0.21 & $96.4 \pm 2.4$ & $96.2 \pm 2.2$ & 0.4 \\
\hline *Independent t test & & & & & &
\end{tabular}

No significant difference was observed between the mean of respiratory rate, $\mathrm{SPO}_{2}$ in the experimental and control group at 30 minutes after music therapy $(\mathrm{p}<0.2)$. (Table 3$)$. 
Table 3 Comparing mean of physiological parameters in two groups

\begin{tabular}{lcccccc}
\hline Groups & \multicolumn{3}{c}{ Before intervention } & \multicolumn{3}{c}{ After intervention } \\
\hline Physiological parameters & $\begin{array}{c}\text { Experimental } \\
\text { group }\end{array}$ & Control group & p-value* & $\begin{array}{c}\text { Experimental } \\
\text { group }\end{array}$ & Control group & p-value* \\
\hline Systolic blood pressure & $140.1 \pm 3.18$ & $150.2 \pm 2.8$ & 0.6 & $120.2 \pm 2.1$ & $145.3 \pm 1.2$ & 0.04 \\
Diastolic blood pressure & $110.2 \pm 0.28$ & $95.2 \pm 1.4$ & 0.54 & $100.2 \pm 0.8$ & $92.1 \pm 1.8$ & 0.08 \\
Pulse rate & $110.1 \pm 2.3$ & $115.1 \pm 4.2$ & 0.5 & $82.3 \pm 2.2$ & $1.8 .2 \pm 3.2$ & 0.03 \\
Respiratory rate & $24.2 \pm 1.2$ & $28.2 \pm 3.1$ & 0.4 & $22.3 \pm 1.8$ & $24.2 \pm 2.2$ & 0.2 \\
$\mathrm{SPO}_{2}$ & $96.3 \pm 2.2$ & $96.4 \pm 2.4$ & 0.5 & $97.7 \pm 2.1$ & $96.2 \pm 2.2$ & 0.03 \\
\hline
\end{tabular}

* Paired $\mathrm{t}$ test

\section{Discussion}

Most of the using methods for reducing the postoperative complications such as; pain and the amount of analgesic drugs consumption are based on drugs intervention. Our effort in this study was to assess the effect of music as a non pharmacological and inexpensive intervention on postoperative physiological parameters. Based on the results of this study, music significantly difference postoperative systolic blood pressure, pulse rate in patients after open heart surgery. Like our study, Smolen et al showed that music effected on changes in physiological parameters [19]. Chlan pointed out that music therapy would lower heart rate and systolic blood pressure by making comfortable feeling and relaxation in patients that related to Adrenalin level of blood [20]. Some of the studies indicated that music therapy would reduce blood pressure and pulse rate, but no changes were observed in respiratory rate [21-25]. The results of Nilsson study showed music therapy would decrease blood pressure, pulse- and respiratory rates [26]. Our findings are compatible with the results of Chan [27], Hatem et al [28], Reza et al [29] and Chlan [20]. No significant difference was observed between the mean of respiratory rate, $\mathrm{SPO}_{2}$ level, diastolic blood pressure in the experimental and control groups before intervention. Yilmaz et al conducted a study to evaluate the effect of music on the hemodynamic parameters in extracorporeal shock wave lithotripsy treatment. The increase in oxygen saturation at the end of treatment was statistically significant in the music group. The commonly accepted theory explaining the pain-, anxiety-, and stress-reducing effects of music is that music acts as a distracter, focusing the patient's attention away from negative stimuli to something pleasant and encouraging. Therefore, patients' vital signs could be stabilized and $\mathrm{SPO}_{2}$ increase [30]. The findings from the present study support earlier research and literature findings that suggest that music increases $\mathrm{SPO}_{2}$ values in the participants. The finding that listening to music was effective in postoperative heart rate, Systolic Blood Pressure (SBP) is consistent with other studies that examined the effects of music on pain for patients undergoing cardiac surgery [31]. Hamel [32] and Yung et al [33] investigated the effect of music on vital sign after surgery and reported that there are no significant difference in blood pressure level, heart rates after music therapy. This finding is inconsistent with present study. The inconsistencies between different studies may be attributed to differences in Type of music, Music time, Type of disease, Culture patient and Music playback time.Alemrud and Petersson believe that music therapy could effect on brain and stimulate alpha waves which the result is releasing endorphins and vital signs controlled in the following [34].

The current study is consistent with the findings from previous studies that sedative music was more effective than scheduled rest or usual treatment in SBP, heart rate in ICU. Studies that have not found significant effects on physiological parameters [35] or have found mixed results small sample sizes with inadequate power to detect significant differences [35], when patients may have had difficulty focusing on the music intervention Experts have suggested that sedative music is more effective if the patient is able to concentrate on the intervention [35]. It seems that sedative music used in this study, would be effective in decreasing the patients' pain after surgery.

Music ineffectiveness on SBP, heart rate in these 
studies could be attributed to the type of the music listened by the patients, i.e. one type of Spanish guitar music was played for all participants without considering the local and national culture of the patients. However, it has been suggested that preferred music, as opposed to prescribed music, is a critical factor in the effectiveness of music therapy [36]. The results of Woldendorp et al. showed a significant correlation between the degree of relaxation and liking the music [37]. Since the emotional responses differ from culture to culture, a type of music related to the cultural features of the subjects should be selected [3] .

The success of music interventions may be greatly enhanced by familiarity and cultural contexts of the patients. The large effects for those who used sedative music may be attributable to having something more pleasant to concentrate on or something to distract their minds from the pain and help them relax their bodies [38]. Some researchers believe that music as the senses deviation acts like a mask on the annoying sounds of the ICU and leads to reduction of stress and anxiety and reduction of the pain intensity in the following [39]. Music also occupies the patient's mind with something familiar and soothing, which allows the patient to escape into his or her own world [36]. This study had several limitations. The study was conducted in only one ICU, and the study sample reflects only one area of Iran. Data collected by one researcher. The available selection of music included only non verbal musical pieces, and this limited participants' choice. Future studies are recommended to include larger samples from different regions in Iran and should also include a larger selection of music.

\section{Conclusion}

Music could be used as a non-invasive tool in order to control physiological parameters following surgery. However, the music listened to by patients should be of a sedative quality, and culturally appropriate selections should be offered. Nurses can use music as an intervention for patients who have undergone open heart surgery to promote nursing autonomy and the idea that nurses are able to affect patients' environment. It is advisable that music become a part of nursing care offered to patients experiencing pain, because music is a low-cost therapy that has no side effects.

\section{Acknowledgements}

The authors would like to thank all patients and physicians who participated in this study, especially Dr. Porabbasi (the cardiovascular specialist) and all nurses in ICU.

\section{Contribution}

Study design: NMA.

Data collection and analysis: AM, HN, SHKH.

Manuscript preparation: NMA, AM, HN, SHKH.

\section{Conflict of Interest}

"The authors declare that they have no competing interests."

\section{Funding}

This paperis the result of a research project granted by Kashan University of Medical Sciences and Health care Services (grant number: 9246). The study was also registered at Iranian registry for clinical trial (IRCT) as IRCT201406028348N7.

\section{Reference}

1- Zand Parsa AF, Ziai H, Haghighi L. The impact of cardiovascular risk factors on the site and extent of coronary artery disease. Cardiovasc J Afr2012; 23(4): 197-9.

2- Allred KD, Byers JF, Sole ML. The effect of music on postoperative pain and anxiety. Pain Manag Nurs2010; 11(1): 15-25.

3- Molavi Vardanjani M, Masoudi Alavi N, Sadat Razavi N, Aghajani M, Azizi-Fini E, Morteza Vaghefi S. A randomized-controlled experimental examining the effects of reflexology on anxiety of patients undergoing coronary angiography. Nurs Midwifery Stud2013; 2(1): 3-9.

4- Choinière $\mathrm{M}$, Watt-Watson $\mathrm{J}$, Victor $\mathrm{JC}$, et al. Prevalence of and risk factors for persistent postoperative nonanginal pain after cardiac surgery: a 2-year prospective multicentre study. Can Med Assoc J2014; 186(7): E213-23.

5- Sabzevari S, Mohammad Alizadeh S, Khodarahmi M. Correlation between preoperative demographic and physiologic variables and extubation time after coronary artery bypass graft (CABG). Iran Journal of Nursing2006; 19(47): 67-77.

6- Arora S, Hull L, Sevdalis N, et al. Fcators compromising safety in surgery: stressful events in the 
operating room. Am J Surg2010; 199(1): 60-5.

7- Yorke J, Wallis M, McLean B. Patient's perceptions of pain management after cardiac surgery in an Australian critical care unit. Heart Lung2004; 33(1): 33-41.

8- Hong SJ, Hong MK, Ko YG, et al. Migration of calcium and atheromatous plaque in computed tomography: an important mechanism of coronary artery occlusion after transcatheter aortic valve replacement. J Am Coll Cardiol2014; 63(12): 23.

9- Jafari H, Emami Zeydi A, Khani S, Esmaeili R, Soleimani A. The effects of listening to preferred music on pain intensity after open heart surgery. Iran $J$ Nurs Midwifery Res2012;17(1):1-6.

10- Renn CL, Dorsey SG. The physiology and processing of pain: a review. AACN Adv Crit Care2005; 16(3): 277-90. 11- Power I. Recent advances in postoperative pain therapy. Br J Anaesth2005; 95(1): 43-51.

12-Hillecke T, Nickel A, Bolay HV. Scientific perspectives on music therapy. Ann N Y Acad Sci2005; 1060: 271-82.

13- Alipour Z, Eskandari N, Ahmari Tehran H, Eshagh Hossaini SK, Sangi S. Effects of music on physiological and behavioral responses of premature infants: a randomized controlled experimental. Complement Ther Clin Pract2013; 19(3): 128-32.

14- Vaajoki A, Kankkunen P, Pietila AM, VehviläinenJulkunen K. Music as a nursing intervention: effects of music listening on blood pressure, heart rate, and respiratory rate in abdominal surgery patients. Nurs Health Sci2011; 13(4): 412-8.

15- Camara JG, Ruszkowski JM, Worak SR. The effect of live classical piano music on the vital signs of patients undergoing ophthalmic surgery. Medscape J Med2008; 10(6): 149.

16- Labrague LJ, McEnroe-Petitte DM. Influence of music on preoperative anxiety and physiologic parameters in women undergoing gynecologic surgery. Clin Nurs Res2016; 25(2): 157-73.

17- Ghetti CM. Effect of music therapy with emotionalapproach coping on preprocedural anxiety in cardiac catheterization: a randomized controlled experimental. $J$ Music Ther2013; 50(2): 93-122.

18- Cruise CJ, Chung F, Yogendran S, Little DA. Music increases satisfaction in elderly outpatients undergoing cataract surgery. Can J Anaesth1997; 44(1): 43-8

19- Smolen D, Topp R, Singer L. The effect of selfselected music during colonoscopy on anxiety, heart rate, and blood pressure. Appl Nurs Res2002; 15(3): 126-36.

20- Chlan L, Halm MA. Does music ease pain and anxiety in the critically ill? Am J Crit Care2013; 22(6): 528-32.

21- Mok E, Wong KY. Effects of music on patient anxiety. AORN J2003; 77(2): 396-7, 401-6, 409-10.

22- Ozer N, Karaman Ozlu Z, Arslan S, Gunes N. Effect of music on postoperative pain and physiologic parameters of patients after open heart surgery. Pain
Manag Nurs2013; 14(1): 20-8.

23- Ferrer AJ. The effect of live music on decreasing anxiety in patients undergoing chemotherapy treatment. J Music Ther2007; 44(3): 242-55.

24- Haibo Yu, Yongfeng Liu, Shuzhen Li, Xiaoming Ma. Effects of music on anxiety and pain in children with cerebral palsy receiving acupuncture: A randomized controlled experimental. Int J Nurs Stud2009; 46(11): 1423-30.

25- Bulfone T, Quattrin R, Zanotti R, Regattin L, Brusaferro S. Effectiveness of music therapy for anxiety reduction in women with breast cancer in chemotherapy treatment. Holist Nurs Pract2009; 23(4): 238-42.

26- Nilsson U. The effect of music intervention in stress response to cardiac surgery in a randomized clinical experimental. Heart Lung2009; 38(3): 201-7.

27- Chan MF. Effects of music on patients undergoing a C-clamp procedure after percutaneous coronary interventions: a randomized controlled experimental. Heart Lung2007; 36(6): 431-9.

28- Hatem TP, Lira PI, Mattos SS. The therapeutic effects of music in children following cardiac surgery. $J$ Pediatr (Rio J)2006; 82(3): 186-92.

29.- Reza N, Ali SM, Saeed K, Abul-Qasim A, Reza TH. The impact of music on postoperative pain and anxiety following cesarean section. Middle East Anesthesiol2007; 19(3); 573-86.

30- Yilmaz E, Ozcan S, Basar M, Basar H, Batislam E, Ferhat M. Music decreases anxiety and provides sedation in extracorporeal shock wave lithotripsy. Urology2003; 61(2): 282-6.

31- Trappe HJ. Role of music in intensive care medicine. Int J Crit Illn Inj Sci2012; 2(1): 27-31.

32- Hamel WJ. The effects of music intervention on anxiety in the patient waiting for cardiac catheterization. Intensive Crit Care Nurs2001; 17(5): 279-85.

33- Yung PM, Chui-Kam S, French P, Chan TM. A controlled experimental of music and pre-operative anxiety in Chinese men undergoing transurethral resection of the prostate. J Adv Nurs2002; 39(4): 352-9. 34- Almerud S, Petersson K. Music therapy--a complementary treatment for mechanically ventilated intensive care patients. Intensive Crit Care Nurs2003; 19(1): 21-30.

35- Good M, Ahn S. Korean and American music reduces pain in Korean women after gynecologic surgery. Pain Manag Nurs2008; 9(3): 96-103.

36- Chlan LL, Engeland WC, Savik K. Does music influence stress in mechanically ventilated patients? Intensive Crit Care Nurs2013; 29(3): 121-7.

37- Woldendorp KH, Van de Werk P, Boonstra AM, Stewart RE, Otten E. Relation between muscle activation pattern and pain: an explorative study in a bassists population. Arch Phys Med 
Rehabil2013;94(6):1095-106.

38- Chen CK, Pei YC, Chen NH, et al. Sedative music facilitates deep sleep in young adults. J Altern Complement Med2014;20(4):312-7.
39- Gelinas C, Arbour C, Michaud C, Robar L, Cote J. Patients and ICU nurses' perspectives of nonpharmacological interventions for pain management. Nurs Crit Care2013;18(6):307-18.

Copyright $(2016$ ASP Ins. This open-access article is published under the terms of the Creative Commons Attribution-NonCommercial 4.0 International License which permits Share (copy and redistribute the material in any medium or format) and Adapt (remix, transform, and build upon the material) under the Attribution-NonCommercial terms. 